\title{
ON THE OVERDETERMINED SYSTEM ABOUT SURFACES WITH PARALLEL MEAN CURVATURE VECTOR FIELD
}

\author{
SHINYA HiRakawa
}

\section{Introduction}

The study of surfaces with parallel mean curvature vector field in complex space forms generalizes the theory of constant mean curvature surfaces in threedimensional Euclidean space. Such surfaces are locally determined, firstly in the case of four-dimensional real space forms, and secondly of two-dimensional complex space forms. In the last case, Ogata [2] got the overdetermined system of the immersions and solved it under the assumption that the Gaussian curvatures of the surfaces are constant. Later, Kenmotsu-Zhou [1] solved it without any additional conditions and determined locally the explicit representations of such immersions. In this paper, we give another method to solve this system of Ogata.

First, we explain the result of [2]. Let $M$ be an oriented and connected twodimensional Riemannian manifold, $X$ a complex two-dimensional complex space form with constant holomorphic sectional curvature $4 \rho$. Let $x: M \rightarrow X$ be an isometric immersion with parallel mean curvature vector field $H$ with $|H|=2 b>$ 0 . And let $\theta$ be the Kähler angle of $x$. Then on $M^{\prime}=\{p \in M \mid \theta(p) \neq 0, \pi\}$ there exist a complex structure $\phi$, a real valued function $a$ and a complex valued function $c$ such that:

$$
\begin{aligned}
d \phi & =\cot \theta \cdot(a-b) \phi \wedge \bar{\phi}, \\
d \theta & =(a+b)(\phi+\bar{\phi}), \\
d a & =\left\{2 \cot \theta \cdot(a-b) a+\frac{3}{4} \rho \sin 2 \theta\right\}(\phi+\bar{\phi}), \\
d c \wedge \bar{\phi} & =2 \cot \theta \cdot(a-b) c \phi \wedge \bar{\phi}, \\
|c|^{2} & =a^{2}+\frac{\rho}{2}\left(3 \sin ^{2} \theta-2\right) .
\end{aligned}
$$

Since $M$ is a real two-dimensional Riemannian manifold, there exists an isothermal coordinate around each point of $M$. When we take the appropriate isothermal coordinate $(U,(u, v)), U \subset M^{\prime}$, we can write $\phi=\lambda(d u+\sqrt{-1} d v)$,

Received November 8, 2001; revised March 25, 2002. 
where $\lambda>0$ is an isothermal factor on $U$. Then the overdetermined system obtained in [2] is written as follows in this coordinate.

THEOREM 1. Under the notations above, there exist an isothermal coordinate $(u, v)$ around each point of $M^{\prime}$ and functions $\lambda, \theta$, a depending only on u-variable, such that they satisfy the following differential equations:

$$
\begin{aligned}
& \frac{d \lambda}{d u}=-2 \lambda^{2} \cot \theta \cdot(a-b), \quad \lambda>0, \\
& \frac{d \theta}{d u}=2 \lambda(a+b), \\
& \frac{d a}{d u}=2 \lambda\left\{2 \cot \theta \cdot(a-b) a+\frac{3}{4} \rho \sin 2 \theta\right\}, \\
& \log \lambda^{4}\left(a^{2}+\frac{\rho}{2}\left(3 \sin ^{2} \theta-2\right)\right)=k_{1} u+k_{2},
\end{aligned}
$$

where $k_{1}$ and $k_{2}$ are some real numbers.

Also, the function $c$ in (4) and (5) can be written as

$$
c=\sqrt{a^{2}+\frac{\rho}{2}\left(3 \sin ^{2} \theta-2\right)} e^{\sqrt{-1}\left(-k_{1} v / 2+t\right)},
$$

where $t$ is a real constant.

In [2], Ogata showed that conversely if we have a simply connected domain $M$ in $(u, v)$-plane and some real valued functions $\lambda, \theta, a$ on $M$ which satisfy the system of differential equations above, then there exists an immersion $x: M \rightarrow X$ such that, with respect to the induced metric, its mean curvature vector field $H$ is parallel with $|H|=2 b>0$ and its Kähler angle is $\theta$. Such an $x$ is uniquely determined up to holomorphic isometric transformations of $X$.

Kenmotsu-Zhou [1] solved this system (1)-(5) as follows:

THEOREM 2. On the Ogata's overdetermined system we have $k_{1}=0$ and the solutions are given by:

$(\alpha)$ When $\rho>0, a$ and $\theta$ must be constants and satisfy

$$
a \equiv-b, \quad \theta \equiv \frac{\pi}{2}
$$

( $\beta$ ) When $\rho=0$ and $a$ is constant, we have $a \equiv 0$ or $a \equiv b$ or $a \equiv-b$. When $\rho=0$ and $a$ is not constant, $\theta$ is determined as a function of $a$ by

$$
\sin ^{2} \theta=c_{1} \frac{(a-b)^{2}}{|a|}
$$

where $c_{1}>0$ is a real constant. 
$(\gamma)$ When $\rho<0$, we have

$$
\begin{aligned}
& a \equiv-b, \quad \theta \equiv \frac{\pi}{2} \quad \text { or } \\
& a \equiv-b, \quad \sin \theta \equiv \sqrt{\frac{8}{9}} \text { or } \\
& a=b\left(1-\frac{9}{4} \sin ^{2} \theta\right) .
\end{aligned}
$$

Remark. In [1] the solutions $a \equiv 0$ and $a \equiv b$ in the case of $\rho=0$ are missed.

In [1], the fact of $k_{1}=0$ is shown by studying the behavior of the solutions of the overdetermined system. Once we prove the fact, it is easy to find all solutions of the system (1)-(5). In this paper we prove $k_{1}=0$ by the different method from [1].

Acknowledgements. The author would like to express his gratitude to Professor Katsuei Kenmotsu for his constant guidance and encouregement during the course of this work. The author would like also to thank the members of the geometry seminar of the Mathematical Institute of Tohoku University for their kind advice.

\section{Another proof of Theorem 2}

We assume $c \neq 0$, and represent $c=|c| e^{\sqrt{-1} \tau}$ on the points of $c \neq 0$, where $\tau$ is a real valued function. We know from (10) that $k_{1}=0$ if and only if $\tau$ is constant. To prove that $\tau$ is constant, we show the following.

THEOREM 3. The solution of the overdetermined system (1)-(5) satisfies either $\theta \equiv \pi / 2$ or $\rho=0$ or $4 a-4 b+9 b \sin ^{2} \theta \equiv 0$.

To prove Theorem 3, we prepare three lemmas.

Lemma 1. Assume $c \not \equiv 0$ and $\theta$ is a constant function. Then it holds that $\theta \equiv \pi / 2$.

Proof. Since $\theta$ is constant, we have $d \theta \equiv 0$. Hence by (2), $a$ is a constant function and it holds that $a \equiv-b$. Then by (5) $|c|$ is a constant function. From this we have $d c=\sqrt{-1} c d \tau$. By (4), we also have $d c \wedge \bar{\phi}=-4 b \cot \theta \cdot c \phi \wedge \bar{\phi}$. Since $c \not \equiv 0$ and $|c|$ is constant, it does not take the value 0 . And since $\tau$ is a real valued function, we have $\sqrt{-1} d \tau=-4 b \cot \theta \cdot(\phi-\bar{\phi})$. By the exterior differentiation of this equation, we obtain $-4 b \cot \theta \cdot(d \phi-d \bar{\phi})=0$. It follows that $\cot \theta=0$ or $d \phi-d \bar{\phi}=0$. Since $d \phi$ is imaginary by (1), the later case also implies $\cot \theta=0$. This proves Lemma 1 . 
Lemma 2. Assume $c \neq \equiv$, and put $c=|c| e^{\sqrt{-1} \tau}$ on the points of $c \neq 0$. Then it holds that

$$
\begin{aligned}
& \sqrt{-1}\left(2 a^{2}+\rho\left(3 \sin ^{2} \theta-2\right)\right) d \tau \\
& \quad=-\rho \cot \theta \cdot\left(9 b \sin ^{2} \theta+4(a-b)\right)(\phi-\bar{\phi}) .
\end{aligned}
$$

Proof. By (2), (3) and (5), we have

$$
\begin{aligned}
d c^{2}=d\left(|c|^{2} e^{\sqrt{-1} 2 \tau}\right) & \\
=e^{\sqrt{-1} 2 \tau}\{ & \left(-4 \cot \theta \cdot(b-a) a^{2}+\frac{3}{2} \rho a \sin 2 \theta\right)(\phi+\bar{\phi}) \\
& \left.+\frac{3}{2} \rho \sin 2 \theta \cdot(a+b)(\phi+\bar{\phi})+\sqrt{-1}\left(2 a^{2}+\rho\left(3 \sin ^{2} \theta-2\right)\right) d \tau\right\} .
\end{aligned}
$$

On the other hand, it follows from (4) that

$$
d c^{2} \wedge \bar{\phi}=4 \cot \theta \cdot(a-b)\left(a^{2}+\frac{\rho}{2}\left(3 \sin ^{2} \theta-2\right)\right) e^{\sqrt{-1} 2 \tau} \phi \wedge \bar{\phi}
$$

Compairing these two equations, we get

$$
\begin{aligned}
& \cot \theta \cdot(a-b)\left(4 a^{2}+2 \rho\left(3 \sin ^{2} \theta-2\right)\right) \phi \wedge \bar{\phi} \\
&=\left\{-4 \cot \theta \cdot(b-a) a^{2}+\frac{3}{2} \rho \sin 2 \theta \cdot(2 a+b)\right\} \phi \wedge \bar{\phi} \\
&+\sqrt{-1}\left(2 a^{2}+\rho\left(3 \sin ^{2} \theta-2\right)\right) d \tau \wedge \bar{\phi} .
\end{aligned}
$$

Simplifying this equation, we see that (11) holds, because $\tau$ is a real valued function. This proves Lemma 2.

Lemma 3. When $\rho=-3 b^{2}$, there exists a solution of the system (1)-(5) such that $c \not \equiv 0$ and $4(a-b)+9 b \sin ^{2} \theta \equiv 0$.

Proof. For a solution $\theta(u)$ of the next differential equation

$$
\frac{d \theta}{d u}=\frac{b}{2} \sqrt{8-9 \sin ^{2} \theta(u)}
$$

with the initial condition $\theta(0)=\theta_{0}, 0<\theta_{0}<\sin ^{-1} \sqrt{8 / 9}$, we define

$$
\begin{aligned}
& \lambda(u)=\frac{1}{\sqrt{8-9 \sin ^{2} \theta(u)}}, \\
& a(u)=b\left(1-\frac{9}{4} \sin ^{2} \theta(u)\right) .
\end{aligned}
$$


Then these functions satisfy the system (6)-(9) with $\rho=-3 b^{2}$. In this case we have $|c|^{2}=b^{2}\left(8-9 \sin ^{2} \theta\right)^{2} / 16$, which is not zero on a neighborhood of $u=0$. This proves Lemma 3 .

Now, we prove Theorem 3. By the exterior differentiation of (11), we have

$$
\begin{aligned}
& \sqrt{-1}\left(4 \cot \theta \cdot(a-b) a^{2}+3 \rho \sin \theta \cos \theta \cdot(2 a+b)\right)(\phi+\bar{\phi}) \wedge d \tau \\
& \quad=\rho\left(-4 a^{2}-9 a b \sin ^{2} \theta+27 b^{2} \cos ^{2} \theta-5 b^{2}+6 \rho \cos ^{2} \theta\right) \phi \wedge \bar{\phi} .
\end{aligned}
$$

From (11) and (12), if $\rho \neq 0$, then putting $x=\sin ^{2} \theta$, we have

$$
\begin{aligned}
& a^{4}(32-24 x)+a^{3}\left(-64 b+136 b x-54 b x^{2}\right) \\
& +a^{2}\left(32 b^{2}-148 b^{2} x+126 b^{2} x^{2}+28 x \rho-24 x^{2} \rho\right) \\
& +a\left(-24 b x \rho+114 b x^{2} \rho-81 b x^{3} \rho\right) \\
& +20 b^{2} x \rho-42 b^{2} x^{2} \rho+27 b^{2} x^{3} \rho+12 x \rho^{2}-30 x^{2} \rho^{2}+18 x^{3} \rho^{2}=0 .
\end{aligned}
$$

We denote by $P_{1}(a, x)$ the polynomial defined by the left hand side of the equation above, where $a$ and $x$ are indeterminates. By (2) and (3) we get

$$
\begin{aligned}
& d a=\cot \theta \cdot\left(2(a-b) a+\frac{3}{2} \rho x\right)(\phi+\bar{\phi}), \\
& d x=\cot \theta \cdot 2 x(a+b)(\phi+\bar{\phi}) .
\end{aligned}
$$

If we assume $\theta \not \equiv \pi / 2$, then the exterior differentiation of $P_{1}(a, x)=0$ gives us the following:

$$
\begin{aligned}
& a^{5}(256-240 x)+a^{4}\left(-640 b+1232 b x-540 b x^{2}\right) \\
& +a^{3}\left(512 b^{2}-1432 b^{2} x+1116 b^{2} x^{2}+360 x \rho-336 x^{2} \rho\right) \\
& +a^{2}\left(-128 b^{3}+296 b^{3} x-440 b x \rho+1296 b x^{2} \rho-891 b x^{3} \rho\right) \\
& +a\left(136 b^{2} x \rho-384 b^{2} x^{2} \rho+216 b^{2} x^{3} \rho+24 x \rho^{2}-36 x^{2} \rho^{2}+36 x^{3} \rho^{2}\right) \\
& +40 b^{3} x \rho-168 b^{3} x^{2} \rho+162 b^{3} x^{3} \rho+24 b x \rho^{2} \\
& -156 b x^{2} \rho^{2}+279 b x^{3} \rho^{2}-\frac{243}{2} b x^{4} \rho^{2}=0 .
\end{aligned}
$$

We denote by $P_{2}(a, x)$ the polynomial defined by the left hand side of this equation, where $a$ and $x$ are indeterminates.

We assume $c \not \equiv 0$ and $\rho \neq 0$ and $\theta \not \equiv \pi / 2$ in what follows. If there exists a solution of the system (1)-(5) in this case, then it must satisfy $P_{1}(a, x)=0$ and $P_{2}(a, x)=0$. Then $P_{1}(a, x)$ and $P_{2}(a, x)$ must have a common factor $f(a, x)$.

We find a necessary condition for $P_{1}(a, x)$ and $P_{2}(a, x)$ to have the common factor $f(a, x)$. If we assume $\partial f / \partial a=0$, then the equation $f(a, x)=0$ is a polynomial equation with respect to $x$ only, and hence $x$ must be a constant function. Then by Lemma 1 , we have $\theta \equiv \pi / 2$, which contradicts the assumption. 
Thus we have $\partial f / \partial a \neq 0$. It follows that if we substitute some number $x=x_{0}$ into $P_{1}(a, x)$ and $P_{2}(a, x)$, then the polynomials $P_{1}\left(a, x_{0}\right)$ and $P_{2}\left(a, x_{0}\right)$ must have the common factor $f\left(a, x_{0}\right)$.

We can factorize $P_{1}(a, 1)$ as follows:

$$
P_{1}(a, 1)=(a+b)(4 a+5 b)\left(2 a^{2}+\rho\right) .
$$

So, if $P_{1}(a, 1)$ and $P_{2}(a, 1)$ have a common factor, then either $(a+b)$ or $(4 a+5 b)$ or $(a \pm \sqrt{-\rho / 2})$ must be a factor of $P_{2}(a, 1) . \quad P_{2}(-b, 1)$ is factorized by

$$
P_{2}(-b, 1)=\frac{1}{2} b\left(2 b^{2}+\rho\right)\left(8 b^{2}+3 \rho\right) .
$$

Therefore, if $P_{2}(a, 1)$ has a factor $(a+b), \rho$ must be $-2 b^{2}$ or $-8 b^{2} / 3$.

Next, we factorize $P_{2}(-5 b / 4,1)$ as follows:

$$
P_{2}\left(-\frac{5 b}{4}, 1\right)=-\frac{9}{16} b\left(3 b^{2}+\rho\right)\left(25 b^{2}+8 \rho\right) .
$$

Then, if $P_{2}(a, 1)$ has a factor $(4 a+5 b), \rho$ must be $-3 b^{2}$ or $-25 b^{2} / 8$.

For the last case, we study $P_{2}(a, 1)$ with $\rho=-2 a^{2}$. It is witten as

$$
P_{2}(a, 1)=2\left(a+\sqrt{-\frac{\rho}{2}}\right)\left(a-\sqrt{-\frac{\rho}{2}}\right) Q(a)+4 a^{2}(a+b)(4 a+5 b)^{2},
$$

where $Q(a)$ is a polynomial of $a$. In this case, to have a common factor, $\rho$ must be 0 or $-2 b^{2}$ or $-25 b^{2} / 8$.

It follows from the discussion above and the assumption $\rho \neq 0$ that $\rho$ must be $-2 b^{2}$ or $-3 b^{2}$ or $-8 b^{2} / 3$ or $-25 b^{2} / 8$.

We observe that $P_{1}(a, x)$ and $P_{2}(a, x)$ have no common factor except the case of $\rho=-3 b^{2}$. To verify this, we need only to show that $P_{1}(a,-1)$ and $P_{2}(a,-1)$ have no common factor when $\rho \neq-3 b^{2}$. In these cases, we may assume $b=1$ because $P_{1}(a,-1)$ and $P_{2}(a,-1)$ are homogeneous polynomials for $a$ and $b$.

To this end, it suffices to prove that they have no common factor over rational field $\boldsymbol{Q}$. For, if $P_{1}(a,-1)$ and $P_{2}(a,-1)$ have a common factor, then they have a common root $\alpha$. Therefore since all their coefficients are rational, the homomorphism $\boldsymbol{Q}[a] \rightarrow \boldsymbol{C}$ which is the identity on $\boldsymbol{Q}$ and maps $a$ on $\alpha$ has a non-zero kernel. In this case the kernel is an ideal which is principal, generated by a single polynomial. Hence $P_{1}(a,-1)$ and $P_{2}(a,-1)$ have a common factor with rational coefficients.

To see that $P_{1}(a,-1)$ and $P_{2}(a,-1)$ have no common factor over $\boldsymbol{Q}$, we show they have no common factor over a prime field $\boldsymbol{F}_{p}$. We substitute $\rho=-2 b^{2}$ and $x=-1$ and $b=1$ into $P_{1}(a, x)$ and $P_{2}(a, x)$, and over $\boldsymbol{F}_{5}$ we have

$$
\begin{aligned}
& P_{1}(a,-1) \equiv a^{4}+a^{3}+2 a+3(\bmod 5), \\
& P_{2}(a,-1) \equiv a^{5}+3 a^{4}+2 a^{3}+2 a^{2}+3 a+3(\bmod 5) .
\end{aligned}
$$


Using Euclidean algorithm, we see that these polynomials have no common factor.

In the cases of $\rho=-8 b^{2} / 3$ and $-25 b^{2} / 8$, we substitute $x=-1$ and $b=1$ into $P_{1}(a, x)$ and $P_{2}(a, x)$, and over $\boldsymbol{F}_{11}$ we have

$$
\begin{aligned}
& P_{1}(a,-1) \equiv a^{4}+10 a^{3}+a^{2}+10 a+5(\bmod 11), \\
& P_{2}(a,-1) \equiv a^{5}+8 a^{4}+10 a^{3}+3 a^{2}+4 a+1(\bmod 11) .
\end{aligned}
$$

We remark $-8 / 3 \equiv(-8+11) / 3 \equiv 1$ and $-25 / 8 \equiv(-25+33) / 8 \equiv 1$ and $1 / 2 \equiv$ $(1+11) / 2 \equiv 6(\bmod 11)$. Using Euclidean algorithm, we see that these polynomials have no common factor.

When $\rho=-3 b^{2}$, we know that there is a solution which satisfies $4(a-b)+$ $9 b \sin ^{2} \theta \equiv 0$ and $|c| \not \equiv 0$ by Lemma 3. In this case, since a common factor of $P_{1}(a, 1)$ and $P_{2}(a, 1)$ is only $(4 a+5 b)$, the common factor of $P_{1}(a, x)$ and $P_{2}(a, x)$ is only $4(a-b)+9 b x$.

As a result, we see that if $c \not \equiv 0$, then we have either $\theta \equiv \pi / 2$ or $\rho=0$ or $4 a-4 b+9 b x \equiv 0$, and moreover we have $d \tau \equiv 0$ by (11). Hence $\tau$ is a constant function, and the function $c$ is written as

$$
c=\sqrt{a^{2}+\frac{\rho}{2}\left(3 \sin ^{2} \theta-2\right)} e^{\sqrt{-1} t},
$$

where $t$ is a real constant.

When $c \equiv 0$, by the exterior differentiation of $|c|^{2} \equiv 0$, we have either $\theta \equiv \pi / 2$ or $\rho=0$ or $4 a-4 b+9 b \sin ^{2} \theta \equiv 0$.

Consequently, any solution of the overdetermined system (1)-(5) satisfies either $\theta \equiv \pi / 2$ or $\rho=0$ or $4 a-4 b+9 b \sin ^{2} \theta \equiv 0$. This completes the proof of Theorem 3 .

In what follows, we find $a$ and $\theta$ in these cases and prove Theorem 2 .

$(\alpha)$ The case of $\theta \equiv \pi / 2$.

In this case $a$ must be a constant function and we get $a \equiv-b$ by (2). Also we have $\rho \geq-2 b^{2}$ because of $|c|^{2} \geq 0$.

( $\beta)$ The case of $\rho=0$.

In this case we have $d a=2 \cot \theta \cdot(a-b) a(\phi+\bar{\phi})$ by (3), and we need to consider two cases separately.

$(\beta-1)$ The case when $a$ is a constant function:

We have $\theta \equiv \pi / 2$ or $a \equiv 0$ or $a \equiv b$. When $\theta \equiv \pi / 2$, we have $a \equiv-b$.

$(\beta$-2) The case when $a$ is not a constant function:

We regard $\theta$ as a function of $a$. Since $d \theta=(d \theta / d a) d a$, we have

$$
(a+b)=\frac{d \theta}{d a} 2 \cot \theta \cdot(a-b) a .
$$

By the integration of this equation, we get 


$$
\sin ^{2} \theta=c_{1} \frac{(a-b)^{2}}{|a|}
$$

where $c_{1}$ is a positive constant.

( $\gamma$ ) The case of $4 a-4 b+9 b \sin ^{2} \theta \equiv 0$.

In this case by the exterior differentiation of this equation, we have $d a=$ $-(9 b / 4) \sin 2 \theta d \theta$. Substituting (2) and (3) into the formulas of $d a$ and $d \theta$ above, we obtain $\rho=-3 b^{2}$. Furthermore, if $\theta$ is a constant function, then we have $a \equiv-b$ and $\sin \theta \equiv \sqrt{8 / 9}$.

We obtained all solutions of the overdetermined system (1)-(5), and proved Theorem 2.

\section{REFERENCES}

[1] K. Kenmotsu And D. Zhou, The classification of the surfaces with parallel mean curvature vector in two-dimensional complex space forms, Amer. J. Math., 122 (2000), 295-317.

[2] T. Ogata, Surfaces with parallel mean curvature vector in $P^{2}(\boldsymbol{C})$, Kodai Math. J., 18 (1995), 397-407.

Mathematical Institute

TOHOKU UNIVERSITY

980-8578 SENDAI, JAPAN

e-mail: s99m28@math.tohoku.ac.jp 